\title{
An online application to measure the dispersion function in AGS
}

\author{
N. Tsoupas, H. Huang, F. Meot, \\ J. Morris, S. Nemesure
}

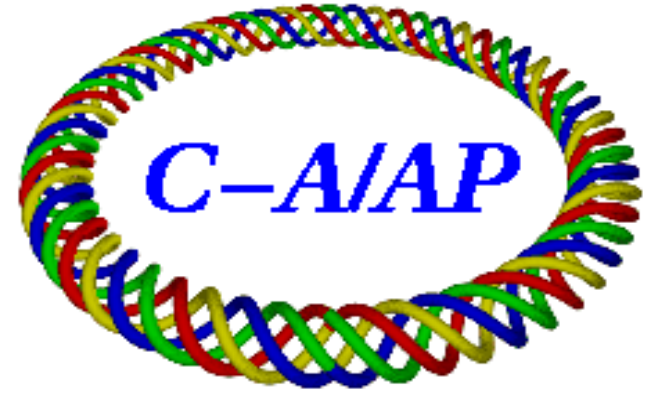

\section{Collider-Accelerator Department Brookhaven National Laboratory Upton, NY 11973}

Notice: This document has been authorized by employees of Brookhaven Science Associates, LLC under Contract No. DE-AC02-98CH10886 with the U.S. Department of Energy. The United States Government retains a nonexclusive, paid-up, irrevocable, world-wide license to publish or reproduce the published form of this document, or allow others to do so, for United States Government purposes. 


\title{
An online application to measure the dispersion function in AGS
}

\author{
Authors: N. Tsoupas, H. Huang, F. Meot, J. Morris, S. Nemesure
}

\begin{abstract}
The measurement of the AGS dispersion function is one of the requirements for the development of an accurate online model of the AGS. Currently the zgoubi and the MADX online models of the AGS are employed as online models to set up and view the beam optics of the AGS using the model viewer. In this technical note we describe the method we employ to measure the numerical values of the dispersion function of the AGS at the location of the Beam Position Monitors (BPM's) and we explain in detail the automatic process to measure experimentally the dispersion function of AGS at the location of the BPM's. This automatic method of measuring the dispersion of the AGS not only reduces dramatically the time required to collect and analyze the experimental data to calculate the dispersion of the AGS, but also minimizes the possible human error that may occur in the process of the dispersion measurement.
\end{abstract}

\section{Definition of Dispersion}

In this technical note we define as dispersion $\left(D_{x}\right)$, the ratio:

$$
D_{x}=\lim _{\frac{p-p_{0}}{p_{0}} \rightarrow 0} \frac{\Delta x}{\left(\frac{p-p_{0}}{p_{0}}\right)}=\lim _{\Delta p \rightarrow 0} \frac{\Delta x}{\left(\frac{\Delta p}{p_{0}}\right)}=\frac{\partial \mathrm{x}}{\partial(\delta p)} \mid x=0 \quad \text { (1). In this definition of the dispersion, } \mathrm{p}_{0} \text { is the }
$$

momentum of the particle along the reference orbit, the symbol $\Delta \mathrm{x}$ is the transverse deviation from the reference orbit of an arbitrary particle having momentum $\mathrm{p}$, and the symbol $\delta \mathrm{p}=\left(\mathrm{p}-\mathrm{p}_{0}\right) / \mathrm{p}_{0}$ is the relative momentum deviation from the reference particle $\mathrm{p}_{0}$. According to this definition if $\mathrm{D}_{\mathrm{x}}=0$ then $\Delta \mathrm{x}=0$ thus the arbitrary particle having momentum (p) moves along the reference orbit when $\mathrm{D}_{\mathrm{x}}=0$.

In a more rigorous but equivalent definition of the dispersion $D_{x}$, we can express the deviation $\Delta x$ of the arbitrary particle with momentum $\mathrm{p}$ as a Taylor series expansion in terms of the relative momentum deviation $\delta \mathrm{p}=\left(\mathrm{p}-\mathrm{p}_{0}\right) / \mathrm{p}_{0}$ as in equation (2) below.

$$
\Delta x(\delta p)=\left.\frac{\partial x}{\partial(\delta p)}\right|_{x=0} \delta p+\left.\frac{1}{2 !} \frac{\partial^{2} x}{\partial^{2}(\delta p)}\right|_{x=0} \delta p^{2}+\left.\frac{1}{3 !} \frac{\partial^{3} x}{\partial^{3}(\delta p)}\right|_{x=0} \delta p^{3}+\ldots
$$

The coefficient of the first term in the Taylor series expansion of function $\Delta \mathrm{x}(\delta \mathrm{p})$ as given by equation (2) is defined as the dispersion $\mathrm{D}_{\mathrm{x}}$.

Thus: $\quad D_{x}=\frac{\Delta x}{\left(\frac{p-p_{0}}{p_{0}}\right)}=\lim _{\Delta p \rightarrow 0} \frac{\Delta x}{\left(\frac{\Delta p}{p_{0}}\right)}=\frac{\partial \mathrm{x}}{\partial(\delta p)} \mid \mathrm{x}=0$

Based on the above definition of dispersion, one can measure the dispersion of a beam at a particular point by measuring the transverse displacement $\Delta \mathrm{x}$ of two particles that have relative momentum difference $\delta=\Delta \mathrm{p} / \mathrm{p}_{0}$ and forming the ratio $\mathrm{D}_{\mathrm{x}}=\Delta \mathrm{x} / \delta$. 


\section{Implementation of the Method}

In this section we discuss the way we apply the definition of the dispersion as given in the previous section, to measure the dispersion of the AGS at the location of the BPM's. All of the tasks are automated and are performed by the computer.

1. We open the "AGS Orbit Control" application and we set the values of the radial function which controls the average beam radius $\bar{r}\left(p_{0}, T 0_{j}\right)$ of the reference orbit or the reference momentum $\left(\mathrm{p}_{0}\right)$ of the centroid of the beam at specific times $\mathrm{T}_{\mathrm{j}}$ during the acceleration cycle. The index $\mathrm{j}$ refers to the number of $\mathrm{T} 0$ times the orbit is measured during a magnet cycle.

2. We run the "AGS Orbit Display" application which measures the position of the centroid of the orbit $\mathrm{x}_{\text {cent }}\left(\mathrm{p}_{0}, \mathrm{TO}_{\mathrm{j}}, \mathrm{BPM}_{\mathrm{i}}\right)$ at the location of the BPM's, and from these positions the application calculates the average radius $\bar{r}\left(p_{0}, T 0_{j}\right)$ of the beam orbit at the various selected times $\mathrm{T} 0_{\mathrm{j}}$ during the acceleration cycle. This average radius $\bar{r}\left(p_{0}, T 0_{j}\right)$ is calculated from the position $\mathrm{x}\left(\mathrm{p}_{0}, \mathrm{TO}_{\mathrm{j}}, \mathrm{BPM}_{\mathrm{i}}\right)$ of the beam's centroid at the location of the BPM's by the use of: $\bar{r}\left(p_{0}, T 0_{j}\right)=\frac{\sum_{i=1}^{n_{B P M}} x_{c e n t}\left(p_{0}, T 0_{j}, B P M_{i}\right)}{n_{B P M}}$ The variable $\mathrm{n}_{\mathrm{BPM}}$ is the number of the BPM's along the AGS ring.

3. With the tasks 1 and 2 we have measured the position $\mathrm{x}_{\mathrm{cent}}\left(\mathrm{p}_{0}, \mathrm{~T}_{0, \mathrm{j}}, \mathrm{BPM}_{\mathrm{i}}\right)$ of the reference orbit $\mathrm{p}_{0}$ at the location of the BPM's and we have calculated the average radius $\bar{r}\left(p_{0}, T 0_{j}\right)$ of the circulating beam at various times $\left(\mathrm{T}_{0, \mathrm{j}}\right)$ during the acceleration cycle. Now we must measure the position of the beam's centroid $\mathrm{x}_{\text {cent }}\left(\mathrm{p}, \mathrm{T0}_{\mathrm{j}}, \mathrm{BPM}_{\mathrm{i}}\right)$ at the location of the BPM's of the arbitrary momentum (p) and subsequently to calculate the average radius $\bar{r}\left(p, T 0_{j}\right)$ of the arbitrary momentum (p). We therefore repeat tasks 1 and 2 above as they are described in tasks 4 and 5 below.

4. We modify the radial function in the "AGS Orbit Control" application to change the average momentum of the beam bunch to the value of the arbitrary momentum (p).

5. The new average momentum (p) will move the centroid of the beam at a new average orbit $\bar{r}\left(p, T 0_{j}\right)$ and the application will provide the location $\mathrm{x}_{\mathrm{cent}}\left(\mathrm{p}, \mathrm{T} 0_{, j}, \mathrm{BPM}_{\mathrm{i}}\right)$ of the centroid of the beam at the location of the BPM's. This average radius $\bar{r}\left(p, T 0_{j}\right)$ of the arbitrary momentum (p) is calculated by the "AGS Orbit Display" application using the formula $\bar{r}\left(p, T 0_{j}\right)=\frac{\sum_{i=1}^{n_{B P M}} x_{c e n t}\left(p, T 0_{j}, B P M_{i}\right)}{n_{B P M}} \quad$ Although in practice these two sets of measurements (tasks 3 and 5) are sufficient to determine the value of the dispersion function at the location of the 
BPM's, we execute step 6 below to calculate the values of the dispersion function with better accuracy.

6. In this task we repeat steps 4 and 5 few more times but each time with a new "arbitrary value of momentum". This provides a set of position values at the location of the BPM's as a function of "arbitrary beam momentum" or "the average radius" of the circulating beam. This is equivalent to generating a function $\Delta \mathrm{x}\left(\delta \mathrm{p}, \mathrm{T}_{\mathrm{j}}, \mathrm{BPM}_{\mathrm{i}}\right)=\mathrm{x}_{\text {cent }}\left(\mathrm{p}, \mathrm{T}_{\mathrm{j}}, \mathrm{BPM}_{\mathrm{i}}\right)-\mathrm{x}_{\text {cent }}\left(\mathrm{p}_{0}, \mathrm{~T}_{\mathrm{j}}, \mathrm{BPM}_{\mathrm{i}}\right)$ which can be expanded as a Taylor series in terms of $\delta p$, as in equation 2 above, to provide the value of the dispersion at the location of the BPM's.

7. To increase the accuracy of the measured dispersion we measure about five orbits (five AGS cycles) for a given average radius (momentum) and we calculate an average centroid of the beam $\bar{X}_{\text {cent }}\left(p_{i}, T 0_{j}, B P M_{k}\right)$ at the location of each BPM using the formula: $\bar{x}_{\text {cent }}\left(p_{i}, T 0_{j}, B P M_{k}\right)=\frac{\sum_{n=1}^{A G S_{\text {_cycles }}} x\left(n, p_{i}, T 0_{j}, B P M_{k}\right)}{A G S_{-} \text {cycles }}$. Subsequently we form the function $\Delta \bar{x}_{\text {cent }}\left(\delta p_{i}, T 0_{j}, B P M_{k}\right)=\bar{x}_{\text {cent }}\left(p_{i}, T 0_{j}, B P M_{k}\right)-\bar{x}_{\text {cent }}\left(p_{0}, T 0_{j}, B P M_{k}\right)$ which can be expanded as a Taylor series in terms of $\delta \mathrm{p}$, as in equation 2 above, to provide the value of the dispersion at the location of the BPM's.

\section{Details in setting up the automatic Dispersion measurement}

In this section we describe in greater details the procedure one has to follow to measure the dispersion of the AGS at the location of the BPM's.

The Application which is used to measure the dispersion of AGS comes under the name: "AgsDispersion" and its source fortran code "AgsDispersion.f” is located in the directory:

/operations/app_store/AgsDispersionMeasure/dispersionCalculation/AgsDispersion/ The "AgsDispersion" can be executed by the command:

/operations/app_store/AgsDispersionMeasure/dispersionCalculation/AgsDispersion/AgsDispersion

The dispersion measurement procedure can be separated in two parts; the data collection-part, and the analysis of the data. Although this separation in two parts is transparent to the user, we describe each part separately hoping to provide more clarity for the reader.

Before the execution of the "AgsDispersion" we should open the "AGS Orbit Display" application to enter the following quantities:

1. Set the active PPM User.

2. Set the times $T_{0}$, during each magnet cycle, at which we want to measure the dispersion.

3. Set the number of orbits for each particular beam radius (usually 5). The measurement of a single orbit requires one magnet cycle. By increasing the number of the measured orbits (number of AGS magnet cycles per orbit) we increase the accuracy in the dispersion measurements, and also we allow for magnet cycles without beam. These magnet cycles without beam are discarded in the calculations. 


\section{The data collection part}

Having set up the "AGS Orbit Display" application, following steps 1 to 3 above, we execute the command:

\section{/operations/app_store/AgsDispersionMeasure/dispersionCalculation/AgsDispersion/AgsDispersion}

The execution of the above command should be done from a directory the user selects to collect the dispersion measurements of the AGS during a "RHIC-run".

Upon the execution of the command above the user is prompted to enter the following parameters:

arg1: Step size in radial steering function. This quantity is in volts and it is the step the beam orbit can be displaced from the previous location.

arg2: Number of steps in each direction. Starting from the original orbit (Reference Orbit) this is the number of orbits with smaller or larger radius to be taken.

arg3: Time to start shift. This is the minimum time $\mathrm{T}_{0}$ during the acceleration cycle the Orbit will move by the step defined in arg1.

arg4: Time to finish shift. This is the maximum time $\mathrm{T}_{0}$ during the acceleration cycle the Orbit will move by the step defined in arg1.

arg5: Active PPM User. The number of the active PPM User (1 through 4)

The above five parameters are used as arguments by the script below:

/operations/app_store/AgsDispersionMeasure/bin/AgsDispersionMeasure.csh arg1, arg2, arg3, arg4, arg5

The function of the script above is to communicate with the following applications:

1. The "AGS Orbit Control" application which controls the beam orbit in the AGS.

2. The "AGS Orbit Display" application which measures the position of the orbit at the location of the BPM's.

An additional function of the script is to write the measured orbits as collected from the "AGS Orbit Display” application on a directory having the generic name: : “arg5_arg3toarg4ms_time-stamp”

The path of this directory is given below:

/operations/app_store/AgsDispersionMeasure/data/arg5_arg3toarg4ms_time-stamp

The directory "arg5_arg3toarg4ms_time-stamp” in the path above, is created each time the script "AgsDispersionMeasure.csh" is executed and we call it self-explanatory because the name of the directory is related to the arguments (arg1 to arg5) as explained above. 
On this directory "arg5_arg3toarg4ms_time-stamp” the filenames of the orbits collected by the "AGS Orbit Display” application are written.

Subsequently the executable code "AgsDispersion” reads the filenames of the orbits from the directory “arg5_arg3toarg4ms_time-stamp” and writes them on to a file "dispersionFrom_AgsOrbitDisplay.in”. This file is created at the directory we execute the code "AgsDispersion" and its content from a particular measurement of the dispersion is shown below.

5 ! Number of Orbit files measured by the "AGS Orbit Display” application; The path and file names are written below

-1.0 !The dispersion will be calculated at the average radius $\mathrm{r}=-1.0 \mathrm{~mm}$ with respect to the radius of the reference orbit which is $0 \mathrm{~mm}$

1.8 ! Conversion factor to convert the Average_Beam_Radius[mm] to [dp/p]

/operations/app_store/AgsDispersionMeasure/data/U4.170to220ms.2013-04-15-12.57.03/BEFOREDISPMEAS.U4.2013-04-15-12.57.03

/operations/app_store/AgsDispersionMeasure/data/U4.170to220ms.2013-04-15-12.57.03/DM.U4.NEG2.S1.2013-04-15-12.57.03

/operations/app_store/AgsDispersionMeasure/data/U4.170to220ms.2013-04-15-12.57.03/DM.U4.NEG2.S2.2013-04-15-12.57.03

/operations/app_store/AgsDispersionMeasure/data/U4.170to220ms.2013-04-15-12.57.03/DM.U4.POS2.S1.2013-04-15-12.57.03

/operations/app_store/AgsDispersionMeasure/data/U4.170to220ms.2013-04-15-12.57.03/DM.U4.POS2.S2.2013-04-15-12.57.03

What follows is an explanation of each line appearing above of the file “dispersionFrom_AgsOrbitDisplay.in”

Line 1: (5) is the number of orbit files as measured by the “AGS Orbit Display” Application

Line 2: (-1) Indicates that the Taylor series expansion will be done about the average radius=-1mm

Line 3: in $\left[\mathrm{mm} /\left(10^{-3} \mathrm{dp} / \mathrm{p}\right)\right]$ Is a conversion factor converting from radius $\Delta \mathrm{R}$ to relative momentum $(\delta)$.

Lines 4 through 8: are the filenames of the orbits collected at various average radii. In this case the first file ( $2^{\text {nd }}$ line) contains the data from the starting orbit and it is usually considered as the reference orbit. The "NEG2" indicates that 2 orbits with radii $-1 \Delta \mathrm{R}$ (“S1") and $-2 \Delta \mathrm{R}$ (“S2") have been collected in the negative direction and the "POS2" indicates that 2 orbits with radii $+1 \Delta \mathrm{R}$ (“S1") and $+2 \Delta \mathrm{R}$ ("S2") have been collected in the positive direction.

Subsequently the executable version of the fortran code “dispersionFrom_AgsOrbitDisplay.f” which calculates the dispersion from the orbit data (positions of orbit at BPM's) reads the files of the orbits from the file "dispersionFrom_AgsOrbitDisplay.in" and calculates the dispersion by using the procedure described in a section below. The computer code "dispersionFrom_AgsOrbitDisplay.f" and its executable version are located in the directory /operations/app_store/AgsDispersionMeasure/dispersionCalculation/source/

The calculated Horizontal and Vertical dispersions at the location of the BPM's for each T0 time are written on files under the names "Hor_Dispersion_xxxx" and "Ver_Dispersion_xxxx". The "xxxx" symbols at the last part of the filenames correspond to the T0 times in msec. The calculated dispersions are also plotted by the use of the plotting program "gnuplot". Figure 1 is an example of plotted dispersion at time T0=572 ms. The user may use the files "Hor_Dispersion_xxxx" and "Ver_Dispersion_xxxx" to re plot the horizontal and vertical dispersions with the plotting program of her choice. 
Timing : $572 \mathrm{~ms}$

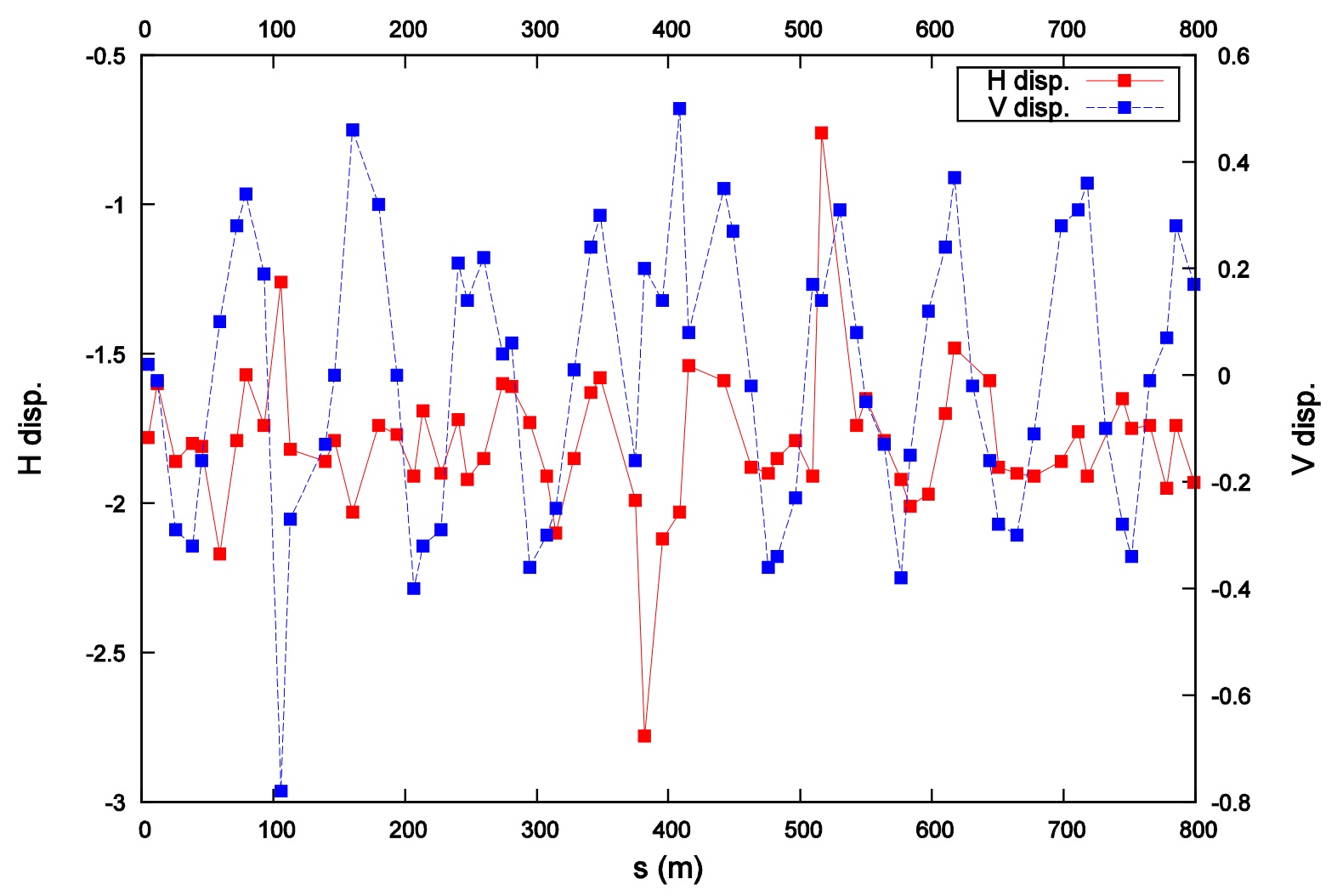

Figure 1. The Horizontal and vertical dispersions at the locations of the AGS BPM's as plotted with the plotting program gnuplot.

\section{Reanalyzing the data}

When reanalysis of the already collected orbits is required, we only use the part of the code which calculates the dispersion from the measured orbits.

In this case we simply execute the command:

/operations/app_store/AgsDispersionMeasure/dispersionCalculation/source/DispersionFrom_AgsOrbitDisplay

We should execute the code above at the same directory where the data had been collected and analyzed at the first time. During the data collection the “dispersionFrom_AgsOrbitDisplay.in" had been created already. This file contains the filenames of the orbit files required for the reanalysis of the data and to generate a measured dispersion from the data. 


\section{Window version of the Application}

A window version of the application which measure the dispersion of the AGS automatically is shown in figure 1 is under "construction". In this window, the values of four of the five arguments (arg1, arg2, arg3, and arg4)) discussed above can be entered on the up right corner of the window. The fifth argument arg5, which is the PPM User can be selected from the PPM menu at the top left corner.

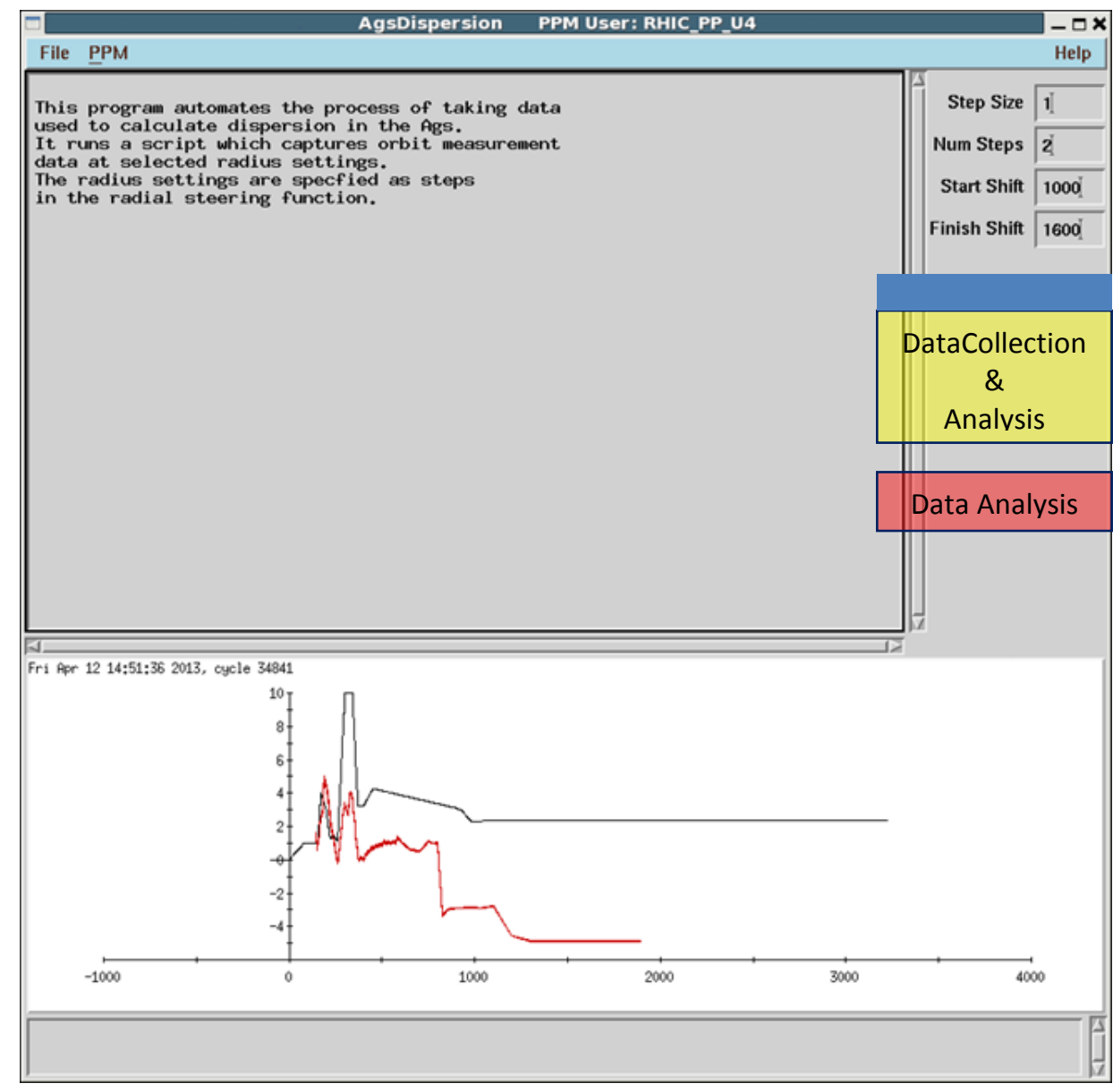

Figure 2. The window of the application for the automatic measurement of dispersion. The values of four of the arguments can be entered in this window, and the PPM user can be entered by clicking on the PPM button at the upper left corner of the window. There will be two buttons of the application window one will enable the user to collect and analyze the data automatically and the other only to analyze the already existing data.

This application window will contain two buttons, one button to collect and analyze the data for the dispersion measurement, and the other button to reanalyze the already collected data. Figure 2 is an example of the application window which is under construction. 


\section{Description of the flowchart of the computer code which calculates the dispersion from the experimental data}

In this section we provide information on the flowchart of the computer code which calculates the dispersion.

Here we assume that we have used the applications "AGS Orbit Control” and "AGS Orbit Display" to measure all the orbits required to calculate the dispersion at the location of the BPM's.

All the information for the calculation of the dispersion is included in the orbit data files which are generated by the “AGS Orbit Display” application.

The code reads from an input file the path and the filenames of the orbit files to be used for the calculation of the dispersion. Each orbit file which corresponds to a given average beam radius contains the following information:

1. The "number of the AGS cycles" which were used to collect each orbit with this average radius.

2. The "number of $\mathrm{T}_{0}$ times" the beam position at the BPM's was measured for this average radius.

3. Sets of the readings of the 72 BPM's which correspond to the horizontal position of the beam's centroid. The number of sets is equal to the "number of the $\mathrm{T}_{0}$ times".

4. Sets of the readings of the 72 BPM's which correspond to the vertical position of the beam's centroid. The number of sets is equal to the "number of the $\mathrm{T}_{0}$ times".

5. Sets of the readings of the 72 BPM's which correspond to the beam current.

The code reads these data from each file and raises a "bad-orbit-flag" for "bad orbits". The "bad orbits" are based on the measured beam intensity (item 5 above) corresponding to each orbit.

Subsequently the code reads each orbit file again to calculate the location of the average beam centroid at the location of each $\mathrm{BPM}_{\mathrm{i}}$, for each beam momentum $\mathrm{p}_{\mathrm{i}}$, and time $\mathrm{T} 0_{\mathrm{j}}$. The set of the $\mathrm{BPM}$ reading with

NO Beam or the readings the bad BPM's are excluded. $\quad \bar{x}_{\text {cent }}\left(p_{i}, T 0_{j}, B P M_{k}\right)=\frac{\sum_{n=1}^{A G S_{\text {ccycles }}} x\left(n, p_{i}, T 0_{j}, B P M_{k}\right)}{A G S_{-} \text {cycles }}$

(4) Location of the beam centroid at the $\mathrm{BPM}_{\mathrm{k}}$

One of these files is the "reference file" and contains the location of the average beam centroid $\bar{x}_{\text {cent }}\left(p_{0}, T 0_{j}, B P M_{k}\right)$ of the average radius of the reference orbit $\bar{r}\left(p_{0}, T 0_{j}\right)$ at the location of the BPM's.

$\bar{x}_{\text {cent }}\left(p_{0}, T 0_{j}, B P M_{k}\right)=\frac{\sum_{n=1}^{A G S \text { cycles }} x\left(n, p_{0}, T 0_{j}, B P M_{k}\right)}{A G S_{-} \text {cycles }}$

The reference momentum $\mathrm{p}_{0}$, and the arbitrary momenta $\mathrm{p}_{\mathrm{i}}$, are proportional to the "Average radius". This “Average radius" is calculated from the summation of the average beam position $\bar{x}_{\text {centr }}\left(p_{i}, T 0_{j}, B P M_{k}\right)$ at the location of the BPM's as in the 
formula. $\quad \bar{r}_{i}\left(p_{i}, T 0_{j}\right)=\frac{\sum_{k=1}^{n_{B P M}} \bar{x}_{c e n t}\left(p_{i}, T 0_{j}, B P M_{k}\right)}{n_{B P M}}$

In the expressions $(4,5)$ the $\mathrm{BPM}_{\mathrm{k}}$, corresponds to any of the 72 BPM's of the AGS which provides valid beam position readings. The $\mathrm{n}_{\mathrm{BPM}}$ in expression (6) is the total number of the BPM's which provide valid beam locations.

Now the code has calculated for each time $\mathrm{T} 0_{\mathrm{j}}$ the average beam position $\bar{x}_{\text {cent }}\left(p_{i}, T 0_{j}, B P M_{k}\right)$ at each BPM as a function of the average beam radius $\bar{r}\left(p_{i}, T 0_{j}\right)$. Since the average beam radius is proportional to the beam momentum we can expand the average beam position $\bar{X}_{\text {cent }}\left(p_{i}, T 0_{j}, B P M_{k}\right)$ as a function of the beam momentum $\mathrm{p}_{\mathrm{i}}$ or $\delta \mathrm{p}=\left(\mathrm{p}-\mathrm{p}_{0}\right) / \mathrm{p}_{0}$ as in equation (2). More specifically we expand the function $\Delta \bar{x}_{\text {cent }}\left(\delta p_{i}, T 0_{j}, B P M_{k}\right)=\bar{x}_{\text {cent }}\left(p_{i}, T 0_{j}, B P M_{k}\right)-\bar{x}_{\text {cent }}\left(p_{0}, T 0_{j}, B P M_{k}\right)$ as a Taylor series in terms of $\delta \mathrm{p}_{\mathrm{i}}=\left(\mathrm{p}_{\mathrm{i}}-\mathrm{p}_{0}\right) / \mathrm{p}_{0}$

The dispersion $\mathrm{D}_{\mathrm{x}}$ at the location of the $\mathrm{BPM}_{\mathrm{i}}$, is the coefficient of the first term of equation (2)

\section{Conclusions}

An automatic computer code has been implemented to measure the dispersion function of the AGS at the location of the BPM's. The results of the measured dispersion function can be used to test the online computer codes zgoubi and MADX which model the beam optics of the AGS. This automatic method of measuring the dispersion of the AGS not only reduces dramatically the time required to collect and analyze the experimental data to calculate the dispersion of the AGS, but also minimizes the possible human error that may occur in the process of the dispersion measurement. 